\title{
Ancienne culture, nouvelle culture (1919)
}

\section{György Lukács}

Gábor Tverdota (éd.)

Traducteur : Gábor Tverdota

\section{(2) OpenEdition}

\section{Journals}

Édition électronique

URL : http://journals.openedition.org/grm/551

DOI : $10.4000 / \mathrm{grm} .551$

ISSN : 1775-3902

Éditeur

Groupe de Recherches Matérialistes

Référence électronique

György Lukács, «Ancienne culture, nouvelle culture (1919)», Cahiers du GRM [En ligne], 6 | 2014, mis en ligne le 23 décembre 2014, consulté le 19 avril 2019. URL : http://journals.openedition.org/grm/551 ; DOI : 10.4000/grm.551

Ce document a été généré automatiquement le 19 avril 2019

(c) GRM - Association 


\section{Ancienne culture, nouvelle culture (1919)}

\section{György Lukács}

Gábor Tverdota (éd.)

Traduction : Gábor Tverdota

\section{NOTE DE L'ÉDITEUR}

Traduit, annoté et présenté par Gabor Tverdota.

Nos remerciements chaleureux vont à Noëmie Charrié et à Alain Loute, qui n'ont pas ménagé leur peine pour améliorer ces traductions par leur relecture attentive et leurs suggestions précieuses.

Nous remercions M. Zoltán Mosóczi pour nous avoir permis la publication de la traduction française de ce texte.

Première parution : Internationale, le 15 juin 1919. La traduction est faite à partir de « Régi kultúra, új kultúra », In : György Lukács, Történelem és osztálytudat [" Histoire et conscience de classe »], Budapest, Magvető, 1971, pp. 29-47 (N.d.t.).

\section{[Comment la culture est-elle possible ?]}

1 L'évolution de la société est un processus unitaire. Cela signifie qu'une certaine phase de cette évolution, à un point donné de la vie sociale, ne peut se dérouler sans que ses effets se répercutent sur tous les autres points. C'est ce caractère unitaire et cohérent de l'évolution sociale qui permet de décrire le même processus, tantôt du point de vue d'un phénomène, tantôt du point de vue d'un autre - tout en aboutissant toujours à la compréhension du même processus. C'est pourquoi il est possible de parler de la culture comme de quelque chose d'apparemment isolé par rapport aux autres phénomènes sociaux. Car si nous saisissons adéquatement la culture d'une certaine époque, nous 
saisissons d'emblée les racines de l'évolution d'ensemble de cette époque, et nous arrivons aux mêmes résultats que si nous étions partis de l'analyse des rapports économiques.

C'est la culture que les pleureuses de l'ordre social capitaliste effondré ont, encore avant l'effondrement, le plus bruyamment pleurée; elles ont fait passer le souci de leurs intérêts de classe pour le souci des valeurs éternelles de la culture. Au contraire, l'idée qui constitue le point de départ du raisonnement qui va suivre est que la culture de l'époque capitaliste s'est effondrée d'elle-même, avant même qu'advienne l'effondrement économique et politique. Ainsi - contrairement à cette crainte largement répandue - c'est précisément dans l'intérêt de la culture que la longue agonie de l'ordre social capitaliste devait enfin arriver à terme, pour que s'ouvre la voie à une nouvelle culture.

3 Si l'on appréhende la culture de deux époques du point de vue de la science, alors la question qui se pose en premier lieu est de savoir quelles sont les préconditions sociales et économiques de la subsistance de la culture. De cette relation découle la réponse à la question par laquelle nous aurions dû commencer, à savoir : qu'est-ce que la culture à proprement parler? Pour le résumer brièvement : le concept de culture (contrairement à celui de civilisation) renferme en lui tous ces produits et activités de grande valeur qui sont négligeables du point de vue de l'autoconservation immédiate. Par exemple: la beauté intérieure et extérieure d'une maison appartient au concept de la culture, contrairement à sa solidité, à sa capacité d'être chauffée, etc. Et si l'on se pose la question des conditions de possibilité sociales de la culture, sous quel ordre social la culture fleurit, la réponse doit être la suivante : dans une société où les besoins vitaux primaires sont assouvis de telle sorte que leur satisfaction ne demande pas un labeur si lourd qu'il absorbe les énergies vitales. Une société, donc, dans laquelle la culture a des énergies libres à sa disposition.

Il en découle que toute ancienne culture était la culture des classes opprimantes. Seules les classes opprimantes se trouvaient dans la situation de pouvoir mettre toutes leurs plus hautes facultés au service de la culture, abstraction faite de tous les soucis de la subsistance matérielle. Le capitalisme, ici comme ailleurs, a révolutionné l'ensemble de l'ordre social. En abolissant les prérogatives liées aux états ${ }^{1}$, il a également aboli les prérogatives culturelles de la société d'états. Car le capitalisme a mis au service de la production aussi bien la classe dominante, la bourgeoisie. L'un des traits distinctifs essentiels de la société capitaliste par rapport aux sociétés antérieures est qu'en elle la classe exploitatrice elle-même est soumise au procès de production; elle-même est obligée de consacrer ses forces à la lutte pour le profit, de manière analogue à l'ouvrier qui, lui, y est obligé afin d'assurer sa subsistance. (Exemple: le directeur d'usine et, face à lui, le propriétaire terrien au temps du servage.) Cette affirmation peut sembler contredite par ce grand nombre d'oisifs que la classe des capitalistes a produit en son sein et auxquels elle a fait don d'un mode de vie aisé. Mais ici aussi, il faut éviter que notre attention ne soit distraite par des phénomènes de surface. Depuis toujours, pour la culture, seules les meilleures forces des classes dominantes entrent en ligne de compte. Les époques antérieures au capitalisme avaient placées celles-ci dans des rapports sociaux où elles pouvaient mettre leurs aptitudes au service de la culture. En revanche, ce sont précisément ces forces-là dont le capitalisme a fait des esclaves de la production, de même que des ouvriers, même si la compensation matérielle de l'esclavage fut bien différente dans les deux cas.

5 La libération du joug du capitalisme signifie la libération du joug de la domination de la vie économique. La civilisation réalise la domination de l'homme sur la nature, mais en retour elle le rend esclave des mêmes instruments grâce auxquels il lui avait été donné de 
maîtriser la nature. Le capitalisme signale l'apogée de cet esclavage. Dans le capitalisme il n'y a aucune classe qui, en vertu de sa position dans la production, serait apte à produire de la culture. C'est précisément ici que la destruction du capitalisme, la société communiste, saisit le problème. Elle s'efforce de créer un ordre social dans lequel tous partageront le mode de vie qui était celui des classes opprimantes dans les époques d'avant le capitalisme; une situation qui ne pouvait être celle d'aucune classe sous le capitalisme.

Ce n'est qu'avec cela que commence véritablement l'histoire de l'humanité. De même que l'histoire au sens ancien débute avec la civilisation, reléguant ainsi les combats de l'homme contre la nature dans la période "préhistorique ", de même l'historiographie des temps à venir fera-t-elle débuter la véritable histoire de l'humanité avec le communisme pleinement développé. Et la domination de la civilisation ne figurera que comme le second temps « d'avant l'histoire».

\section{[Sur le devenir-marchandise des productions culturelles et leur effet sur la possibilité de la culture]}

7 En conséquence, l'ordre social capitaliste se distinguait avant tout par le fait qu'en lui la vie économique cessait d'être un instrument du fonctionnement vital de la société : la vie économique se décala vers son centre, elle devint la fin de toute activité sociale. La première et plus importante conséquence fut que la vie de la société se transforma en un grand rapport d'échange, que le tout de la société apparut sous la forme d'un grand marché. Au niveau des divers fonctionnements vitaux cela se manifestait de la manière suivante: tout produit de l'ère capitaliste, de même que toute énergie productive ou créatrice, revêtit la forme de la marchandise. Toute chose cessa d'être précieuse par ellemême, en vertu de ses valeurs intrinsèques (par exemple artistiques, morales, etc.), et n'eut de valeur qu'en tant que marchandise à vendre et à acheter sur le marché. Quelles conséquences dévastatrices cela eut sur toutes sortes de cultures - que celles-ci se manifestent sous la forme d'activités, de création d'œuvres ou d'institutions -, il n'est peut-être pas nécessaire de l'analyser ici en profondeur. Dès lors que l'indépendance de l'homme et de la culture vis-à-vis des soucis de l'auto-conservation, l'usage libre et désintéressé de ses forces, sont les préconditions humaines et sociales de la culture, toute chose créée par la culture ne peut véritablement être une valeur culturelle que si cette valeur lui est intrinsèque. À partir du moment où elle revêt un caractère de marchandise, où elle intègre la relation dans laquelle elle se mue en marchandise, le caractère de fin-ensoi a disparu, la possibilité de la culture a disparu.

8 Mais il est également un autre point sur lequel le capitalisme s'en est pris aux racines vitales des possibilités sociales de la culture. Ce point est le rapport de cette dernière à la création des produits. Nous avons vu que du point de vue des produits, la culture ne pouvait exister qu'en tant que fin-en-soi. Et, du point de vue de la relation entre produit et créateur, la culture n'est possible que si, du point de vue de son créateur, la création de chaque produit est un processus unitaire et achevé. Plus précisément : un processus dont les conditions dépendent des possibilités et des aptitudes de l'homme créateur. L'exemple le plus caractéristique d'un tel processus est l'œuvre d'art, à savoir le cas où la réalisation de toute l'œuvre est exclusivement le résultat du travail de l'artiste, et où chaque détail de l'œuvre réalisée est déterminé par les traits individuels de l'artiste. Dans les époques 
précapitalistes, cet esprit artistique régnait sur l'ensemble de l'artisanat. Essentiellement, la production du livre dans l'imprimerie différait aussi peu de son écriture que la peinture d'un tableau différait de la confection d'une chaise (si du moins l'on considère la chose à partir de la nature humaine de la création). La production de type capitaliste en revanche non seulement dépossède l'ouvrier de la propriété des instruments de production, mais, par le biais de la spécialisation croissante dans la division du travail de plus en plus poussée, elle segmente le processus d'élaboration du produit en des parties dont aucune ne réalise une chose qui aurait un sens en elle-même, qui serait achevée en tant que telle. Il n'y a désormais plus aucun ouvrier dont le travail serait en rapport direct et sensible avec le produit final ; ce produit n'a de sens que pour le calcul abstrait du capitaliste, pour qui il n'est que marchandise. Le développement de l'industrie mécanique ne peut qu'accentuer l'inhumanité de ce rapport. En effet, tandis que dans le cas de la division du travail qui se crée au sein de la manufacture, aussi poussé que puisse être par ailleurs le degré de segmentation du processus de production, la qualité des divers travaux partiels demeure encore indissolublement liée aux possibilités physiques et mentales de l'ouvrier, dans le cas de l'industrie hautement mécanisée même ce lien entre produit et producteur se trouve aboli: désormais ce sont exclusivement les possibilités de la machine qui dictent le processus de la production; l'homme se met au service de la machine, il s'adapte à la machine; la production devient complètement indépendante des possibilités et des capacités humaines de l'ouvrier².

9 À côté de ces forces détruisant la culture, que nous avons envisagées jusqu'à présent du seul point de vue des produits isolés et de leurs producteurs, d'autres forces semblables sont également opérantes dans le capitalisme. Nous en apercevons la plus importante avec clarté si nous observons les rapports mutuels entre les produits élaborés. La culture des époques précapitalistes était rendue possible par le rapport de continuité dans lequel se trouvaient les divers produits culturels: dans les uns se développaient les questions posées dans les autres, etc. Par-là, la culture montrait les signes d'une certaine continuité de l'évolution lente et organique. Cela rendait possible l'émergence d'une culture en tous points cohérente, unanime, mais tout de même originale; une culture dont le niveau outrepassait de loin le niveau le plus élevé atteignable par les capacités des individus pris isolément. En révolutionnant - comme chacun le sait - le processus de la production, en perpétuant par le biais de l'anarchie productive cet aspect révolutionnaire de la production, le capitalisme a aboli cette continuité et cette organicité des cultures anciennes. Car, du point de vue de la culture, la révolution de la production signifie d'un côté que le processus de production amène sans cesse à la surface des circonstances qui influent de manière décisive sur le cours de la production et sur son comment, sans qu'elles soient dans un rapport quelconque avec l'essence du produit lui-même, ce dernier étant entendu comme œuvre qui trouve sa fin en elle-même. (C'est ainsi que la matérialité dépérit dans toute l'industrie, dans l'architecture, etc.) D'un autre côté, en conséquence du fait que c'est pour le marché que l'on produit - sans quoi le caractère révolutionnaire de la production capitaliste serait inimaginable -, ce sont la pure nouveauté, les éléments surprenants, tapageurs, qui se font valoir dans l'élaboration du produit, sans aucune considération pour le problème de la valeur véritable, intérieure, du produit, sans égards pour le fait que celui-ci s'en trouve rehaussé ou rabaissé. Le reflet culturel de ce caractère révolutionnaire de la production est le phénomène que nous avons coutume d'appeler la mode. Or, mode et culture se réfèrent à deux concepts qui, de manière essentielle, s'excluent mutuellement. Le règne de la mode signifie que la forme, la qualité, etc., des produits lancés sur le marché se transforment en de courts intervalles 
de temps, sans aucune considération pour la question de savoir si, du point de vue de l'efficacité ou de la beauté, elles ont fait ou non leurs preuves. C'est la nature même d'un tel marché qui entraîne la conséquence selon laquelle dans des intervalles de temps donnés de nouvelles choses doivent voir le jour, et notamment des choses nouvelles qui sont radicalement différentes des anciennes, qui ne doivent nullement se fonder sur les expériences acquises au cours de l'élaboration des anciennes. En raison de la rapidité de l'évolution, soit il s'avère tout simplement impossible de recueillir et de digérer ces expériences, soit personne ne veut de toute manière s'appuyer sur elles, puisque la nature de la mode exige précisément quelque chose de radicalement différent de l'ancien. Aussi toute évolution organique se voit-elle détruite, et c'est une précipitation sans but fixe, ainsi qu'un amateurisme prétentieux et fanfaron qui vient prendre sa place.

\section{[La crise de la culture en régime capitaliste : sur la contradiction entre l'idéologie comme forme et l'ordre de la production comme contenu de la culture]}

10 Mais les racines de la crise de la culture capitaliste sont encore plus profondes que les phénomènes ainsi esquissés ne le laisseraient penser. La cause ultime de sa crise permanente et de son effondrement interne est que l'idéologie et l'ordre de la production, l'ordre social, entrent dans une opposition insurmontable. Comme conséquence nécessaire de l'anarchie productive, la classe bourgeoise en lutte pour le pouvoir, puis dominante, ne pouvait avoir qu'une seule idéologie que l'on pouvait prendre au sérieux : l'idéologie de la liberté individuelle. Or, la crise de la culture capitaliste devait intervenir dès le moment où cette idéologie s'est trouvée en opposition avec l'ordre social bourgeois. Dans la période où - ce fut le cas au XVIII siècle - la bourgeoisie ascendante avait opposé cette idéologie aux contraintes de la société d'états, cette idéologie était l'expression précise de la situation donnée de la lutte des classes. La bourgeoisie de cette époque pouvait dès lors réellement se targuer d'être une couche détenant une culture véritable. Cependant, dès que la bourgeoisie a accédé au pouvoir (c'est-à-dire déjà au cours de la Révolution Française), il s'est révélé que la réalisation opiniâtre de cette idéologie, son application sur le tout de la société, étaient impossibles sans que l'ordre social, dont a résulté l'idée de la liberté individuelle comme sa propre idéologie, ne s'anéantisse lui-même. Bref : il était impossible pour la classe bourgeoise d'appliquer sa propre idée de liberté au prolétariat. Le caractère insurmontablement pervers de cette situation s'exprimait selon la formule suivante : soit la bourgeoisie devait renoncer à cette idéologie, soit elle devait en user comme d'un voile dissimulant des actions qui étaient contraires à ses propres préceptes. Dans le premier cas, c'est une absence totale d'idéaux, une abjection pure qui s'en sont suivies, parce que la bourgeoisie, en raison de sa position dans le procès de production, s'est avérée incapable d'engendrer une idéologie autre que celle de la liberté individuelle. Dans le second cas, la bourgeoisie se trouvait face à la faillite morale du mensonge interne : elle se voyait réduite à agir sans cesse contre sa propre idéologie.

11 Cette crise fut encore exacerbée en raison de la faillite économique du principe de la liberté. Il n'est pas possible d'entamer ici une analyse de l'époque du capital financier. Il suffit de pointer le fait que l'aspect hautement organisé de la production (les cartels, les trusts) qui caractérise cette époque est dans une contradiction irréductible avec l'idée dominante de l'ordre économique du capitalisme précoce : la libre concurrence. Avec cela, l'idéologie 
qui naquit dans son sillage perdit tous ses fondements. C'est comme cela que la bourgeoisie - dont les couches supérieures devinrent, de par les conséquences nécessaires du capitalisme financier, les alliées naturelles de leurs anciens adversaires, les classes féodales-agraires -, dut aller chercher une idéologie chez ses nouveaux alliés. Cependant, pas plus que les précédentes, cette tentative - harmoniser l'idéologie avec l'ordre de la production - ne pouvait être fructueuse. Car pour ce qui concerne les fondements véritables des idéologies conservatrices - la structuration en états et l'ordre de la production qui y correspond -, c'est précisément le caractère révolutionnaire de la production de type capitaliste qui les a extirpés de la société. Certes, le féodalisme possédait jadis une haute culture d'une valeur élevée. Mais c'était au temps où la société féodale, d'états, était dominante; lorsque ses principes gouvernaient l'ensemble de la société. Avec la montée au pouvoir du capitalisme, cette forme de société a été anéantie. Ce fut en vain qu'une bonne partie du pouvoir politique, économique et social était restée entre les mains des états jadis dominants. Le processus commandant leur capitalisation, l'assomption par eux-mêmes des formes du capitalisme, ne pouvaient être refoulés. Et par là s'imposa à ces couches également la même perversion de la contradiction entre l'idéologie et l'ordre de la production qui s'imposa au capitalisme, même si la contradiction se manifestait d'une manière différente. Ainsi donc, lorsqu'à l'époque du capital financier la bourgeoisie partait à la recherche de la source de la régénération, elle la trouvait au fond d'un puits qu'elle avait elle-même veillé, auparavant, à condamner.

$\mathrm{Du}$ point de vue de la culture, cette contradiction entre idéologie et ordre de la production signifie la chose suivante : la grandeur des anciennes cultures (la grécité, la Renaissance, etc.) trouve sa cause dans l'harmonie entre l'idéologie et l'ordre de la production de manière à ce que les produits de la culture puissent croître de manière organique, à partir du socle de la vie sociale. Aussi supérieures qu'aient été les grandes œuvres par rapport à la sensibilité de l'homme moyen de l'époque, un certain accord existait tout de même entre elles. Mais, encore plus décisif que cela - l'insertion des produits de la culture au sein de la vie sociale -, l'harmonie entre l'idéologie et l'ordre de la production rendait possible à son tour, d'une manière qui allait de soi, l'harmonie entre l'idéologie et le mode de vie. (Que la qualité du mode vie des hommes est la fonction de la place que ces derniers occupent dans la production, cela, je l'espère, ne nécessite pas de plus amples explications.) Or, dans tout ordre social où le mode de vie et son expression idéologique sont naturels, où ils sont en harmonie d'une manière qui va de soi, l'idéologie peut s'incarner organiquement dans le développement-même de la culture. Cette unité organique n'est possible que dans de telles conditions, car la relative indépendance des éléments idéologiques vis-à-vis de la base économique signifie qu'en tant que formes de manifestations humaines, elles sont indépendantes des données qu'elles informent, données qui leurs sont destinées par l'ordre socio-économique de leur époque en tant que ce-qui-doit-être-mis-en-forme. Mais la matière informée par ces formes ne peut être autre que la réalité sociale elle-même. Donc, si une opposition essentielle émerge entre idéologie et ordre de la production, alors de notre point de vue cette opposition doit être saisie par la tournure suivante : c'est la forme et la matière des manifestations culturelles qui sont entrées en contradiction. Et par là est abolie l'unité organique des œuvres de la culture, est aboli pour saisir cette même situation à partir de la perspective des hommes qui se rapportent à la culture - ce caractère harmonique, jouissif, de la culture, qui caractérisait en propre les cultures anciennes. La culture du capitalisme, dans la mesure où elle était honnête, ne pouvait être autre chose qu'une critique impitoyable de l'époque capitaliste. Cette critique a souvent pu atteindre un niveau très élevé (Zola, Ibsen), mais plus elle était honnête, 
élevée, plus elle devait manquer de l'harmonie simple, naturelle, de la beauté des cultures anciennes: c'est-à-dire de la culture au sens propre, véridique du terme. Et cette contradiction était présente dans tous les champs possibles des manifestations humaines, dans l'ensemble du champ matériel de la culture. Ainsi, pour ne prendre qu'un exemple très frappant, l'ordre capitaliste a nécessairement donné naissance - dans le cadre de son idéologie de la liberté - à l'idée que l'homme est une fin-en-soi. Nous pouvons dire en toute confiance que dans les époques précapitalistes, cette grande idée n'avait jamais reçue une expression aussi pure, claire et consciente que dans ces temps-là (par exemple, l'idéalisme allemand, Kant et Fichte). Mais précisément, et par opposition à cela, jamais aucun ordre social n'a autant foulé au pied cette idée que ne le fit la société capitaliste. Le devenir marchandise de toute chose dans le capitalisme ne se confina pas au devenir marchandise de tous les produits. Il gagna également les rapports humains; il suffit de penser à l'évolution du mariage, le plus important parmi tous les rapports humains, pour que cette situation apparaisse avec clarté. Ainsi, d'un côté, la nécessité intérieure de cette tendance idéologique, culturelle, posait l'exigence envers tous les produits de la culture de diffuser cette idée de l'homme comme fin en soi. Cependant, la matière informée par ces formes culturelles était la négation directe de cette idée. La grande poésie du capitalisme, par exemple, ne pouvait en conséquence pas être la simple représentation de son époque - comme c'était le cas, je présume, de la poésie grecque, dont la beauté immortelle émanait précisément du caractère exempte de critique et allant de soi de cette représentation -, mais pouvait seulement être la critique de la condition existante.

Dans la mesure où des tentatives de représenter les conditions existantes d'une manière simple eurent lieu, cette contradiction entre forme et contenu, entre forme et matière, a bel et bien été supprimée, mais une contradiction encore plus profonde, dissolvant la culture d'une manière encore plus radicale, prit alors la place de la précédente: la contradiction au sein de la forme. Car la représentation sans critique de l'existence ne pouvait émerger qu'au prix de la négation, du masquage, conscient ou inconscient, des aspects pervers de la condition réelle. Il s'agit là d'une source d'où nul art véritable, nulle science véritable, nulle culture véritable ne peut émaner. De là qu’à nulle autre époque il n'y eut une telle quantité et une telle extension de mauvais art et de pseudosciences qu'à l'époque du capitalisme.

\section{[Les préalables pour la résolution de la crise de la culture en régime communiste : sur le changement de fonction de l'idéologie et de l'économique]}

14 Considérons maintenant ce que signifie la transformation de la société dans l'esprit du communisme du point de vue de la culture. Elle signifie avant tout l'abolition de la domination exercée par la vie économique sur la vie dans son ensemble. Elle signifie par conséquent la suppression du rapport pervers et impossible entre l'homme et son travail dans lequel c'est l'homme qui est soumis aux moyens de la production et non le moyen à l'homme. En dernière analyse, cette situation sociale signifie l'abolition du caractère de fin-en-soi de la vie économique. L'ordre social capitaliste a planté si profondément sa structure dans la pensée de chacun, que peu sont à même de prendre conscience avec une clarté suffisante de cet aspect-là des changements. D'autant plus qu'au niveau des phénomènes superficiels de la vie cette partie de la mutation n'a pas encore pu trouver d'expression. Et pourtant, la maitrise de la vie économique, l'organisation socialiste de la vie 
économique signifie la suppression de l'autonomie de la vie économique. La vie économique, qui jusque-là était un processus autonome, avec des lois propres, que la raison humaine ne pouvait que reconnaître, mais pas commander ${ }^{3}$, devient désormais une partie de l'administration publique de l'État, une partie d'un processus planifié unitaire, non régi par ses lois propres. Car les mobiles ultimes de ce processus social unitaire ne sont désormais plus de nature économique. Certes, ici encore les apparences vont à l'encontre de ce constat. Car il est évident que tant théoriquement que pratiquement, la réorganisation de la production est irréalisable autrement que sur une base économique, avec des organismes économiques, guidée par des raisonnements économiques. Il va de soi en outre qu'en conséquence de la nature de la lutte des classes dans la phase de la dictature [du prolétariat, $n$. d.t.] - qui marque l'apogée de la lutte des classes - la question de la réorganisation de l'économie est la question qui se trouve à l'avant-scène. Mais cela ne veut absolument pas dire que les racines ultimes du processus qui se déroule de la sorte seraient également économiques. Le changement de fonction impliqué par la dictature du prolétariat dans tous les domaines est aussi bien à l'œuvre ici. Alors que dans les crises de la société capitaliste tout élément idéologique n'était que la «superstructure» de ce processus révolutionnaire qui a finalement mené à l'effondrement du capitalisme, maintenant cette situation se renverse. Je ne dis pas que c'est la réorganisation de l'économie qui devient "superstructure " (cette expression, même du point de vue de l'idéologie, n'est pas la plus heureuse, parce qu'elle se prête à une série de malentendus), mais on peut dire en revanche qu'elle a perdu sa qualité de première importance. Ce qui, à la surface, s'oppose à ce constat, vient à son renfort, dès que l'on appréhende la situation ne fût-ce qu'avec un point de vue dialectique minimal. Dans les crises de la société capitaliste, c'était toujours la partie idéologique qui se tenait à l'avant-scène de la conscience. Non pas par hasard, mais en conséquence de la nécessité selon laquelle les mobiles ultimes de l'évolution ne peuvent jamais devenir tout à fait conscients dans les masses qu'ils mobilisent. La " critique » exercée par le socialisme à l'égard de ces crises et de ces révolutions était de l'ordre du démasquement : elle pointait les mobiles véritables, ultimes, c'est-à-dire le processus économique. Avec la chute du capitalisme, quoi de plus naturel que le point de vue jusqu'à présent « critique » vienne désormais occuper l'avantscène de la création? La question demeure pourtant de savoir si ce changement de fonction n'a pas supprimé le caractère de motif « ultime » qui était le sien dans son rôle précédent. Qu'il l'ait en effet supprimé ressort déjà de ce qui précède. Car le motif économique comme motif ultime ne peut valoir qu'à condition que l'ensemble de la production soit inorganisé. Seules les forces motrices de la production inorganisée peuvent fonctionner sur le modèle des forces de la nature, comme forces aveugles, et ce n'est qu'en tant que telles qu'elles peuvent être les motifs ultimes de toute chose; ou bien tout élément idéologique va s'aligner à ce processus créé par la production inorganisée (il sera superstructure) ou bien il y opposera une résistance sans efficacité. C'est pour cela que dans la société capitaliste tout facteur non-économique n'est que pure idéologie. L'unique exception est la critique socialiste de l'ordre social capitaliste. Car celle-ci n'est pas un corollaire idéologique approbateur ou contestataire de processus particuliers, mais le démasquement de la totalité : un démasquement de l'ensemble du processus économique, lequel démasquement est en même temps une action efficace pour transformer ce processus. Mais ce qui ainsi se transforme, ce n'est pas uniquement l'inorganisation en tant que telle, mais, du même coup, le caractère de fin-en-soi de la vie économique, en dernière instance la direction de celle-ci par des motifs économiques. Du fait que la vie économique s'organise dans l'esprit du socialisme, la direction est saisie par 
les motifs qui, jusqu'à présent, pouvaient prétendre tout au plus à un statut de phénomènes corollaires : la domination de la vie intérieure et extérieure de l'homme par des motifs humains et non-économiques. Nous ne pouvons désormais plus être surpris par le fait que dans de telles conditions la transformation de la vie économique se tient davantage à l'avant-plan de la conscience révolutionnaire que l'élément idéologique qui la meut en dernière instance. Ce changement de fonction dans les consciences se réalise nécessairement avec la victoire du prolétariat. Il s'agit là même d'une continuation directe de la lutte des classes élevée à hauteur de conscience dans les masses du prolétariat; et l'essentiel de la conscience de classe a de tout temps consisté dans le devenir-conscient des intérêts économiques. Le passage au travail constructif - dont ce changement de fonction est l'épisode ultime - ne concerne d'ailleurs pas la conscience de l'intérêt de classe immédiat; dans ce dernier le changement de fonction est pour ainsi dire « subconscient ». Seule la conscience de classe pleine qui, au-delà de l'intérêt direct, s'éveille à la mission du prolétariat dans l'histoire universelle, élève ce motif, ce changement de fonction, jusqu'à la conscience de classe du prolétariat ${ }^{4}$.

C'est ce changement de fonction qui instaure la possibilité de la nouvelle culture. Car la culture est la maîtrise interne de l'homme sur son milieu, tandis que la civilisation signifie la maîtrise externe sur son milieu. De même que la civilisation a créé les instruments de cette maîtrise sur la nature, de même la culture prolétarienne va-t-elle créer les instruments de maîtrise sur la société, dans laquelle, justement, la civilisation - en sa forme la plus évoluée, le capitalisme - avait développé l'esclavage de l'homme vis-à-vis de la société, la production, la vie économique.

16 Le caractère de fin-en-soi de l'homme est la précondition sociologique de la culture. Cette précondition, qui était donnée aux classes dominantes des sociétés précapitalistes, et que le capitalisme avait confisquée à tout le monde, la dernière phase de la victoire du prolétariat la rendra à tout un chacun. Cette transformation, qui signifie le renversement radical de la structure d'ensemble de la société, concerne bien entendu tous les phénomènes dont nous avons fait mention lors de l'analyse des effets destructeurs du capitalisme sur la culture.

Aussi le caractère révolutionnaire et bouleversant de la production sera-t-il aboli avec l'organisation de la production. À la place de la succession anarchique engendrée par la conjoncture, que nous avons coutume d'appeler la mode, viendra se substituer la continuité organique, le progrès véritable. Un progrès où chaque épisode découle de manière nécessaire des préconditions matérielles de l'épisode précédent. Où, donc, tout moment nouveau apporte des solutions aux questions qui sont restées non-résolues dans le précédent, et en même temps pose des questions à résoudre au prochain moment à venir. La conséquence culturelle nécessaire d'une telle évolution découlant de l'essence des choses (et non de la conjoncture) est que le niveau de la culture dépasse à nouveau les capacités des individus isolés. L'association réciproque au travail d'autrui, la continuation mutuelle du travail de chacun par autrui - l'autre précondition sociologique de la culture - devient à nouveau possible. À cela contribue le fait qu'aussi bien les produits de la culture que les relations humaines perdent leur caractère de marchandise. L'abolition du rapport marchand rend son aspect de fin-en-soi à tout ce qui, sous le joug du capitalisme, apparaissait exclusivement ou avant tout comme moment d'un rapport économique. Et la possibilité de la culture se fonde, comme on le sait, sur le fait de s'assurer que la plus grande part possible des formes de manifestations de la vie humaine acquière, de manière toujours plus profonde et plus forte, un caractère de fin-en-soi, ou, ce qui est équivalent : qu'elle en 
vienne à servir l'humanité de l'homme. Car ces deux formes d'être en-vue-de-soi ne s'excluent pas, au contraire, elles sont au service l'une de l'autre et se complètent mutuellement. Si un certain produit (maison, meuble, etc.) est élaboré non pas en tant que marchandise, mais de façon à intensifier au maximum ses propres possibilités d'être beau, alors cela signifie la même chose que si l'on disait que la maison, le meuble, etc., sont au service de l'humanité de l'homme, en s'adaptant aux exigences de celle-ci. Ce n'est donc pas un processus économique indépendant de toute exigence humaine qui les produit, un processus dans lequel ces produits ne figurent que comme marchandises abstraites, et les hommes comme vendeurs et consommateurs tout aussi abstraits. Simultanément, la spécialisation malsaine propre au capitalisme doit également disparaître. Dès que ce n'est plus l'effort abstrait en vue de la vente et de l'achat sur le marché qui détermine la qualité productive des hommes, mais bien le processus devenu unitaire, et mobilisant l'homme dans son entièreté, de la production et de la jouissance du produit en vue de lui-même, alors la spécialisation traverse elle aussi un changement de fonction. Ce n'est pas seulement sa qualité de classe, son caractère de prérogative qui sont supprimés dans la révolution prolétarienne, mais en même temps sa qualité d'étrangeté vis-à-vis de la vie humaine. Avec la réalisation du caractère de fin-en-soi des produits, ceux-ci se rattachent involontairement à la totalité de la vie humaine, aux questions ultimes de la vie humaine. Avec la suppression de l'isolement, de l'individualisme anarchique de l'homme, la société humaine forme un tout organique unitaire tant au niveau des individus qu'au niveau des produits, un tout dont les parties, se soutenant et se complétant les unes les autres, servent toutes l'idée de la supériorité de l'homme.

\section{[L'homme comme fin-en-soi : la culture communiste comme accomplissement libre des principes de la philosophie de l'idéalisme allemand]}

Avec cet objectif, nous sommes arrivés à l'essence de la question. Si le but de la nouvelle société n'était que l'augmentation de la réussite, du bien-être des hommes, alors tous ces changements de fonction n'adviendraient pas ou, plutôt, leur portée passerait presque inaperçue. Alors la tâche de l'État prolétarien pourrait se borner à l'organisation de la production et de la redistribution, et la vie économique - avec, certes, des buts modifiés continuerait à dominer l'idée de l'homme. Alors, naturellement, l'évolution arriverait à terme bien plus rapidement que de cette manière. Car alors, avec l'organisation juste de la production et de la redistribution, l'évolution aurait atteint son but, alors qu'ainsi la réorganisation en question n'a fait que réaliser la possibilité impérissable de parvenir au but. Or, la réalisation elle-même demeure l'objet d'une lutte à part que l'humanité doit encore livrer.

Cependant, la réorganisation de la vie économique en est une précondition inévitable, et pas uniquement en vertu des causes sociologiques que nous venons d'énumérer ni en vertu du fait que seuls des hommes heureux seraient capables de culture. La raison en est bien plutôt cette structure spécifique de la conscience humaine qui veut que les soucis et les misères immédiats, malgré leur bassesse comparative par rapport aux questions ultimes de l'existence humaine, à l'exception des hommes les plus extraordinaires, masquent ces questions ultimes dans la conscience humaine, ne les laissant pas affleurer 
à la surface. Pour éclairer la situation avec un exemple très simple: supposons que quelqu'un est en train de se creuser la tête sur une grande découverte scientifique et qu'un terrible mal de dent le saisisse; il est certain que, dans la plupart des cas, il sera incapable de continuer la réflexion jusqu'à ce que le mal immédiat ne soit soulagé. L'annihilation du capitalisme, la réorganisation sociale de la production, signifient pour l'ensemble de l'humanité la guérison de tous les maux de dents. Disparait de la conscience des hommes tout ce qui les empêche de vivre pour les questions vraiment essentielles: leur conscience est désormais ouverte à l'essence. Mais l'exemple éclaire également les limites de la transformation. Il est certain qu'afin que le travail de l'esprit puisse être repris, le mal de dent doit cesser, mais il est tout aussi sûr qu'une fois le mal de dent parti, la reprise du travail ne s'opérera pas d'elle-même, de manière automatique. Il faut alors un nouvel effort, une nouvelle attitude, un nouvel élan. L'humanité œuvrant n'est pas non plus arrivée au but, si toutes ses misères économiques sont supprimées, tout au contraire, elle n'aura fait que fonder la possibilité d'entamer avec des forces renouvelées le chemin vers son but véritable. La culture est l'incarnation de l'idée de l'homme. La culture est donc faite par les hommes, et non pas par les circonstances. Toute transformation de la société n'est donc que cadre, n'est que possibilité pour l'auto-action, pour la force créatrice spontanée des hommes libres.

C'est pourquoi la sociologie doit s'arrêter à l'analyse des cadres. Quelle sera, du point de vue de son contenu, de son essence, la culture prolétarienne, seules les forces productives en passe de se libérer la détermineront, faute de quoi toute tentative de prédiction apparaîtrait ridicule. Ce que l'analyse sociologique a pu accomplir n'était rien de plus que de montrer que cette possibilité-là, la société prolétarienne l'a réalisée - et que ce n'est précisément que sa possibilité qui a été réalisée. Toute articulation plus poussée dépasse le cercle des investigations scientifiques qui sont ici possibles; tout au plus pourrait-on s'interroger sur les valeurs culturelles qu'une société nouvelle peut reprendre de l'ancienne et les continuer. Car l'idée de l'homme comme fin-en-soi, l'idée de base de la nouvelle culture, est l'héritage de l'idéalisme classique du XIX ${ }^{e}$ siècle. La véritable contribution de l'époque du capitalisme à la construction de l'avenir consiste à créer, sur les ruines mêmes de son effondrement, les possibilités fondamentales de la construction de l'avenir. De la même manière que le capitalisme a créé les préconditions économiques de sa propre annihilation, de la même manière dont il crée l'armature spirituelle écrasante de sa critique par l'esprit prolétarien (rapport de Marx à Ricardo), de la même manière il crée ici encore, dans la philosophie de Kant et de Fichte, l'idée de cette société nouvelle qui est appelée par nécessité à amener sa propre suppression.

\section{NOTES}

1. Ou „ordres" («Stände »). (N. d. t.)

2. Certains lient ce processus à la division du travail au sein de l'industrie et présentent la chose comme si, à cause de cela, elle [la division du travail] devait persister également au-delà de la chute du capitalisme. La question ne peut être exposée ici, je me borne seulement à mentionner que Marx lui-même voyait la chose autrement. Il pensait que l'organisation du travail social et la 
division du travail à l'intérieur de l'industrie étaient inversement proportionnelles : dans une société où l'une s'est développée, l'autre régresse, et vice-versa (cf. Misère de la philosophie). (Note de Lukács).

3. L'économie politique en tant que science autonome est le pendant de cette situation. Avant que celle-ci ne se soit développée, il n'existait pas de science de l'économie au sens moderne - et avec la disparition de celle-ci, celle-là devra également disparaitre. C'est pure idéologie capitaliste que de voir dans les lois de l'économie politique des lois éternelles de la nature. (Note de Lukács).

4. J'ai écrit de manière plus détaillée sur les deux sortes de conscience de classe dans mon article intitulé «La question de la direction intellectuelle et les "ouvriers intellectuels" ", paru dans Internationale, $5^{\mathrm{e}}$ numéro (note de Lukács). 